\title{
FIXED POINTS AND STABILITY OF A CLASS OF NONLINEAR DELAY INTEGRO-DIFFERENTIAL EQUATIONS WITH VARIABLE DELAYS
}

\author{
Hocine Gabsi, Abdelouaheb Ardjouni and Ahcene Djoudi
}

\begin{abstract}
In this work we study a class of second order nonlinear delay integrodifferential equations

$$
\begin{gathered}
\ddot{x}(t)+f(t, x(t), \dot{x}(t)) \dot{x}(t)+\sum_{j=1}^{N} \int_{t-\tau_{j}(t)}^{t} a_{j}(t, s) g_{j}(s, x(s)) d s \\
+\sum_{j=1}^{N} b_{j}(t) x^{\prime}\left(t-\tau_{j}(t)\right)=0,
\end{gathered}
$$

with variable delays and give some new conditions ensuring that the zero solution is asymptotically stable by means of the fixed point theory. Our work extends and improves previous results in the literature such as, D. Pi $[17,18]$ and T. A. Burton [10]. An example is given to illustrate our claim.
\end{abstract}

Keywords: Fixed points, Stability, Delay integro-differential equations, Variable delays.

\section{Introduction}

Time-delay systems constitute basic mathematical models of real phenomena such as nuclear reactors, chemical engineering systems, biological systems, and population dynamics models. Such systems are often sources of instability and degradation in control performance in many control problems. For more than 100 years, the Lyapunov direct method has been the ultimate key tool to study stability problems. The direct method was widely used to study the stability of solutions of ordinary differential equations and functional differential equations. Nevertheless, the pointwise nature of this method and the construction of the Lyapunov functionals have been, and still are, an arduous task (see [7]). Recently, many authors have realized that the fixed points theory can be used to study the stability of the solution (see

Received October 07, 2016.; accepted December 20, 2016.

2010 Mathematics Subject Classification. 34K20, 34K30, 34K40. 
[1]-[11], [14], [16]-[18]). Levin and Nohel [15] studied the global asymptotic stability of a class of nonlinear systems

$$
\ddot{x}+h(t, x, \dot{x}) \dot{x}+f(x)=a(t) .
$$

They obtained asymptotic stability by constructing a proper Lyapunov function. Burton [10] considered the following delay equation

$$
\ddot{x}+f(t, x, \dot{x}) \dot{x}+b(t) g(x(t-L))=0,
$$

where $L$ is a positive constant. By using the fixed point theory, he gave sufficient conditions for each solution $x(t)$ to satisfy $(x(t), \dot{x}(t)) \longrightarrow 0$. D. Pi $[16,18]$ studied the asymptotic stability of two following equations with delays

$$
\begin{gathered}
\ddot{x}+f(t, x, \dot{x}) \dot{x}+b(t) g(x(t-r(t)))=0, \\
\ddot{x}+f(t, x, \dot{x}) \dot{x}+\sum_{j=1}^{N} \int_{t-\tau_{j}(t)}^{t} a_{j}(t, s) g_{j}(s, x(s)) d s=0 .
\end{gathered}
$$

Many other interesting results on fixed points and stability properties can be found in the references therein. In this paper, we consider the equation

$$
\begin{gathered}
\ddot{x}+f(t, x, \dot{x}) \dot{x}+\sum_{j=1}^{N} \int_{t-\tau_{j}(t)}^{t} a_{j}(t, s) g_{j}(s, x(s)) d s \\
+\sum_{j=1}^{N} b_{j}(t) x^{\prime}\left(t-\tau_{j}(t)\right)=0,
\end{gathered}
$$

for $t \geq 0$, where functions $\tau_{j}: \mathbb{R}^{+} \longrightarrow \mathbb{R}^{+}, a_{j}(\cdot, \cdot): \mathbb{R}^{+} \times\left[-\tau_{j}(0), \infty\right) \longrightarrow \mathbb{R}$, $f: \mathbb{R}^{+} \times \mathbb{R} \times \mathbb{R} \longrightarrow \mathbb{R}^{+}, b_{j}: \mathbb{R}^{+} \longrightarrow \mathbb{R}$ and $g_{j}(\cdot, \cdot):\left[-\tau_{j}(0), \infty\right) \times \mathbb{R}^{+} \longrightarrow \mathbb{R}$ are all continuous functions.

The use of ordinary and partial differential equations to model physical or biological systems and processes has a long history, dating to Lotka and Volterra. The main motivation of time delay is due to the fact that all processes take time delays to complete. The delays can represent gestation times, incubation periods, or transport delays. In many cases time delays can be substantial such as gestation and maturation or can represent little lags such as acceleration and deceleration in physical processes. A simple example in nature is reforestation. A cut of forest, after replanting, will take at least 20 years before reaching any kind of maturity. For certain species of trees (redwoods for example) it would be much longer. Hence, any mathematical model of forest harvesting and regeneration clearly must have time delays built into it. Therefore, it become natural to include time delay terms into the differential equations that model population dynamics. The models that incorporate such delay times are referred as delay differential equation models. 
Equation (1.5) is obviously nonlinear. This is due to the presence of the terms $f(t, x, \dot{x}) \dot{x}$ and $g_{j}(s, x(s))$ and the fact that the functions $f$ and $g_{j}$ are arbitraries non necessarily linears. Further, (1.5) is of time delay type. That is, notice that in (1.5) if we let $t=0$ one can obtain

$$
\begin{gathered}
\ddot{x}(0)+f(0, x(0), \dot{x}(0)) \dot{x}(0)+\sum_{j=1}^{N} \int_{-\tau_{j}(0)}^{0} a_{j}(0, s) g_{j}(s, x(s)) d s \\
+\sum_{j=1}^{N} b_{j}(0) x^{\prime}\left(\tau_{j}(0)\right)=0 .
\end{gathered}
$$

Thus, (1.5) requires an initial function condition defined on at least on $\left[-\tau_{j}(0), 0\right]$. Then we must ask that the solution $x(t)$ of (1.5) depends on a delay function and is such that $x(t)=\psi(t), t \in\left[-\tau_{j}(0), 0\right]$. If we do not stipulate that $t-\tau_{j}(t)$ is bounded below, then we must ask that $\psi:(-\infty, 0] \rightarrow \mathbb{R}$ so that $x(t)=\psi(t)$, $t \in(-\infty, 0]$. Then we solve (1.5) for $t \geq 0$ with a the infinite delay function $\tau_{j}(\cdot)$ which is arbitrary continuous function non necessarily constant.

We assume that

$$
\tau_{j}^{\prime}(t) \neq 1 \text { for all } t \geq 0
$$

and

$$
t-\tau_{j}(t) \longrightarrow \infty \text { as } t \longrightarrow \infty, j=\overline{1, N} .
$$

For each $t_{0} \geq 0$, define $m_{j}\left(t_{0}\right)=: \inf \left\{s-\tau_{j}(s): s \geq t_{0}\right\}, j=\overline{1, N}$ and let $m\left(t_{0}\right)=$ $\min \left\{m_{j}\left(t_{0}\right), j=\overline{1, N}\right\}$. Let $\mathcal{C}\left(t_{0}\right):=\mathcal{C}\left(\left[m\left(t_{0}\right), t_{0}\right], \mathbb{R}\right)$ be the space of continuous functions endowed with function supremum norm $\|\cdot\|$, that is, for $\psi \in \mathcal{C}\left(t_{0}\right),\|\psi\|:=$ $\sup \left\{|\psi(s)|: m\left(t_{0}\right) \leq s \leq t_{0}\right\}$. We will also use $\|\varphi\|:=\sup \left\{|\varphi(s)|: s \in\left[m\left(t_{0}\right), \infty\right)\right\}$ to express the supremum of continuous bounded functions on $\left[m\left(t_{0}\right), \infty\right)$ later. It is well known (see [13]) that, for a given continuous function $\psi$ and a number $y_{0}$, there exists a solution for equation (1.5) on an interval $\left[m\left(t_{0}\right), T\right.$ ), and if the solution remains bounded, then $T=\infty$. We denote by $(x(t), y(t))$ the solution $\left(x\left(t, t_{0}, \psi\right), y\left(t, t_{0}, y_{0}\right)\right)$.

Denote by $A(t):=f((t, x(t), y(t))$. We can rewrite equation (1.5) as

$$
\left\{\begin{aligned}
\dot{x}(t)= & y(t) \\
\dot{y}(t)= & -A(t) y(t)-\sum_{j=1}^{N} \int_{t-\tau_{j}(t)}^{t} a_{j}(t, s) g_{j}(s, x(s)) d s \\
& -\sum_{j=1}^{N} \omega_{j}(t) \frac{d}{d t} x\left(t-\tau_{j}(t)\right)
\end{aligned}\right.
$$

with

$$
\omega_{j}(t)=\frac{b_{j}(t)}{1-\tau_{j}^{\prime}(t)} .
$$


Our purpose is to give a necessary and sufficient condition ensuring that the zero solution of this equation is asymptotically stable. To our knowledge, there are few results about its stability and the studied equation has not been yet considered.

\section{Preliminaries}

Suppose that $f$ is a real or complex-valued function of the variable $t>0$ and $p$ is a real or a complex parameter such that $\operatorname{Re}(p)>0$. We define the Laplace transform (see [19], [12]) of $f$ as

$$
F(p)=\mathfrak{L}(f(t))_{(p)}=\int_{0}^{\infty} e^{-p t} f(t) d t .
$$

We also indicate the Laplace transform (2.1) of power function $t^{\gamma}$ is given by

$$
\mathfrak{L}\left(t^{\gamma}\right)_{(p)}=\int_{0}^{\infty} e^{-p t} t^{\gamma} d t=\frac{\Gamma(\gamma+1)}{p^{\gamma+1}}, \gamma>-1, p>0,
$$

with Gamma function $\Gamma(z)$ is defined by the integral

$$
\Gamma(z)=\int_{0}^{\infty} e^{-t} t^{z-1} d t=\mathfrak{L}\left(t^{z-1}\right)_{(1)},
$$

which converges in the right half of the complex plane $\operatorname{Re}(z)>0$. Now, let $-\infty \leq$ $\alpha<\beta \leq+\infty, \varphi:[\alpha, \beta] \rightarrow \mathbb{R}$ and define for $\lambda \in \mathbb{R}$ the integral

$$
F(\lambda)=\int_{\alpha}^{\beta} e^{-\lambda \varphi(t)} f(t) d t .
$$

We assume that there exists a constant $\lambda_{0}>0$ such that for every $\lambda \geq \lambda_{0}$ we have,

$$
\int_{\alpha}^{\beta} e^{-\lambda \varphi(t)}|f(t)| d t<\infty
$$

The following theorem is crucial to reach our goal.

Theorem 2.1. Let $\varphi:\left[\alpha, \beta\left[\longrightarrow \mathbb{R}\right.\right.$ be a function such that $\varphi$ is of class $\mathcal{C}^{1}, \varphi^{\prime}>0$ on $[\alpha, \beta[$. Assume that $f$ is function continuous at $\alpha$ and $f(\alpha) \neq 0$. Then,

$$
F(\lambda) \sim \frac{f(\alpha)}{\varphi^{\prime}(\alpha)} \frac{1}{\lambda} e^{-\lambda \varphi(\alpha)} \quad(\lambda \longrightarrow+\infty) .
$$

Proof. (a) To begin with, $\varphi(t)=t, \alpha=0$;

$$
F(\lambda)=\int_{0}^{\beta} e^{-\lambda t} f(t) d t
$$

We check that $F(\lambda)$ satisfies the property (2.6). Indeed, since $f$ is continuous at $\alpha=0$, then for any given $\varepsilon>0$, one can choose $\eta$ sufficiently small, such that

$$
|f(t)-f(0)| \leq \varepsilon, \text { for } 0 \leq t \leq \eta \text {. }
$$


Next, we decompose $F(\lambda)$ in the following manner

$$
F(\lambda)=f(0) \int_{0}^{\eta} e^{-\lambda t} d t+\int_{0}^{\eta} e^{-\lambda t}(f(t)-f(0)) d t+\int_{\eta}^{\beta} e^{-\lambda t} f(t) d t .
$$

From (2.9) we can establish the following estimates

$$
\begin{aligned}
\int_{0}^{\eta} e^{-\lambda t} d t & =\frac{1}{\lambda}\left(1-e^{-\lambda \eta}\right), \\
\int_{0}^{\eta} e^{-\lambda t}(f(t)-f(0)) d t & \leq \varepsilon \int_{0}^{\infty} e^{-\lambda t} d t=\frac{\varepsilon}{\lambda} .
\end{aligned}
$$

For $t \geq \eta$ we have $\left(\lambda-\lambda_{0}\right)(t-\eta) \geq 0$. Consequently,

$$
\int_{\eta}^{\beta} e^{-\lambda t} f(t) d t \leq e^{-\eta\left(\lambda-\lambda_{0}\right)} \int_{\eta}^{\beta} e^{-\lambda_{0} t} f(t) d t .
$$

(b) Let us return to the general case. For this purpose, consider the function

$$
g:\left[\alpha, \beta\left[\longrightarrow \left[0, \beta_{0}[, \quad t \longmapsto g(t):=\varphi(t)-\varphi(\alpha),\right.\right.\right.
$$

where $\beta_{0}=\varphi(\beta)-\varphi(\alpha)$. We observe that $g$ is bijective on $[\alpha, \beta[$. Denote the reciprocal function of $g$ by

$$
\psi:\left[0, \beta_{0}[\longrightarrow[\alpha, \beta[, \quad u \longmapsto \psi(u) .\right.
$$

The change of variables $t=\psi(u)$ yields the integral formula

$$
F(\lambda)=e^{-\lambda \varphi(\alpha)} \int_{0}^{\beta_{0}} e^{-\lambda u} f(\psi(u)) \psi^{\prime}(u) d u
$$

We see that

$$
\frac{d \psi(u)}{d t}=\psi^{\prime}(\varphi(t)-\varphi(\alpha)) \varphi^{\prime}(t)=1 \text { and } \psi^{\prime}(0)=\frac{1}{\varphi^{\prime}(\alpha)} .
$$

Define

$$
\tilde{f}(u):=f(\psi(u)) \psi^{\prime}(u) .
$$

Clearly, the function $\tilde{f}$ is continuous at 0 . Moreover,

$$
\tilde{f}(0)=f(\psi(0)) \psi^{\prime}(0)=\frac{f(\alpha)}{\varphi^{\prime}(\alpha)} .
$$


Repeated application of (a) yields

$$
\begin{aligned}
F(\lambda)= & e^{-\lambda \varphi(\alpha)} \tilde{f}(0) \int_{0}^{\eta} e^{-\lambda u} d u+e^{-\lambda \varphi(\alpha)} \int_{0}^{\eta} e^{-\lambda u}(\tilde{f}(u)-\tilde{f}(0)) d u \\
& +e^{-\lambda \varphi(\alpha)} \int_{\eta}^{\beta} e^{-\lambda u} \tilde{f}(u) d u \\
\leq & e^{-\lambda \varphi(\alpha)} \frac{f(\alpha)}{\varphi^{\prime}(\alpha)} \frac{1}{\lambda}\left(1-e^{-\lambda \eta}\right)+\frac{\varepsilon}{\lambda} e^{-\lambda \varphi(\alpha)} \\
& +e^{-\lambda \varphi(\alpha)} e^{-\eta\left(\lambda-\lambda_{0}\right)} \int_{\eta}^{\beta_{0}} e^{-\lambda_{0} u} \tilde{f}(u) d u
\end{aligned}
$$

Stability definitions, fixed point technique and more details on delay differential equations can be found in $([13,7])$.

Definition 2.1. The zero solution of (1.8) is stable if for each $\varepsilon>0$ there exists $\delta=\delta\left(\varepsilon, t_{0}\right)>0$ such that $\left[\psi \in \mathcal{C}\left(t_{0}\right), y_{0} \in \mathbb{R},\|\psi\|+\left|y_{0}\right|<\delta\right]$ implies that $\left|x\left(t, t_{0}, \psi\right)\right|+\left|y\left(t, t_{0}, y_{0}\right)\right|<\varepsilon$ for $t \geq t_{0}$.

Definition 2.2. The zero solution of (1.8) is asymptotically stable if it is stable and there is a $\delta_{1}=\delta_{1}\left(t_{0}\right)>0$ such that $\left[\psi \in \mathcal{C}\left(t_{0}\right), y_{0} \in \mathbb{R},\|\psi\|+\left|y_{0}\right|<\delta_{1}\right]$ implies that $\left|x\left(t, t_{0}, \psi\right)\right|+\left|y\left(t, t_{0}, y_{0}\right)\right| \longrightarrow 0$ as $t \longrightarrow \infty$.

\section{Main Results}

In this section, we will prove Theorem 3.1 and Theorem 3.2 on stability and asymptotic stability respectively, for equation (1.5) by using the fixed point theory. But our equation is nonlinear and has no non trivial edo term so the inversion of that equation needs some preparations. Lemma 3.1 and Lemma 3.2 are the subject of these preparations. We use the variation of parameter and then transform the given equation and in Lemma3.3 we invert it and give the expression of the solutions. The proof of these theorems depends on Theorem 2.1.

Lemma 3.1. Applying the variation of parameters formula to the second equation of (1.8), we get

$$
\begin{aligned}
\dot{x}(t)= & B(t)-\sum_{j=1}^{N} \int_{t_{0}}^{t} e^{-\int_{s}^{t} A(u) d u} \int_{s-\tau_{j}(s)}^{s} a_{j}(s, v) g_{j}(v, x(v)) d v d s \\
& -\sum_{j=1}^{N} \int_{t_{0}}^{t} e^{-\int_{s}^{t} A(u) d u} \omega_{j}(s) \frac{d}{d s} x\left(s-\tau_{j}(s)\right) d s
\end{aligned}
$$

where

$$
B(t):=\dot{x}\left(t_{0}\right) e^{-\int_{t_{0}}^{t} A(u) d u}
$$


Proof. Indeed, multiplying both sides of the second equation of (1.5) by the factor $e^{\int_{t_{0}}^{t} A(u) d u}$ and integrating from $t_{0}$ to any $t \in\left[t_{0}, T\right]$, we obtain

$$
\begin{aligned}
y(t)= & y\left(t_{0}\right) e^{-\int_{t_{0}}^{t} A(v) d v}-\sum_{j=1}^{N} \int_{t_{0}}^{t} e^{-\int_{s}^{t} A(v) d v} \int_{s-\tau_{j}(s)}^{s} a_{j}(s, v) g_{j}(v, x(v)) d v d s \\
& -\sum_{j=1}^{N} \int_{t_{0}}^{t} e^{-\int_{s}^{t} A(v) d v} \omega_{j}(s) \frac{d}{d s} x\left(s-\tau_{j}(s)\right) d s .
\end{aligned}
$$

Substituting $\dot{x}(\cdot)$ into $(3.3)$, we get

$$
\begin{aligned}
\dot{x}(t)= & \dot{x}\left(t_{0}\right) e^{-\int_{t_{0}}^{t} A(v) d v} \\
& -\int_{t_{0}}^{t} e^{-\int_{s}^{t} A(v) d v} \sum_{j=1}^{N} \int_{s-\tau_{j}(s)}^{s} a_{j}(s, v) g_{j}(v, x(v)) d v d s \\
& -\int_{t_{0}}^{t} e^{-\int_{s}^{t} A(v) d v} \sum_{j=1}^{N} \omega_{j}(s) \frac{d}{d s} x\left(s-\tau_{j}(s)\right) d s .
\end{aligned}
$$

Making use of (3.2), we see that this last equation gives (3.1).

Lemma 3.2. The equation

$$
\sigma(t)=-\sum_{j=1}^{N} \int_{t-\tau_{j}(t)}^{t} a_{j}(t, s) g_{j}(s, x(s)) d s,
$$

is equivalent to

$$
\begin{aligned}
\sigma(t)= & \sum_{j=1}^{N} \frac{d}{d t} \int_{t-\tau_{j}(t)}^{t} C_{j}(t, s) g_{j}(s, x(s)) d s \\
& +\sum_{j=1}^{N} C_{j}\left(t, t-\tau_{j}(t)\right)\left(1-\tau_{j}^{\prime}(t)\right) g_{j}\left(t-\tau_{j}(t), x\left(t-\tau_{j}(t)\right)\right)
\end{aligned}
$$

where

$$
C_{j}(t, s)=\int_{t}^{s} a_{j}(u, s) d u \text { and } C_{j}\left(t, t-\tau_{j}(t)\right)=\int_{t}^{t-\tau_{j}(t)} a_{j}\left(u, t-\tau_{j}(t)\right) d u .
$$

Proof. Differentiating the integral term in (3.5), we have

$$
\begin{aligned}
& \frac{d}{d t} \int_{t-\tau_{j}(t)}^{t} C_{j}(t, s) g_{j}(s, x(s)) d s \\
= & \int_{t-\tau_{j}(t)}^{t} \frac{\partial}{\partial t} C_{j}(t, s) g_{j}(s, x(s)) d s+C_{j}(t, t) g_{j}(t, x(t)) \\
& -C_{j}\left(t, t-\tau_{j}(t)\right)\left(1-\tau_{j}^{\prime}(t)\right) g_{j}\left(t-\tau_{j}(t), x\left(t-\tau_{j}(t)\right)\right) .
\end{aligned}
$$


It follows that if $C_{j}(t, t)=0, \frac{\partial C_{j}(t, s)}{\partial t}=-a_{j}(t, s)$, then (3.5) is equivalent to (3.6). The calculation shows that the previous conditions on $C_{j}$ yields

$$
C_{j}(t, s)=\int_{t}^{s} a_{j}(u, s) d u \text { and } C_{j}\left(t, t-\tau_{j}(t)\right)=\int_{t}^{t-\tau_{j}(t)} a_{j}\left(u, t-\tau_{j}(t)\right) d u
$$

Lemma 3.3. Let $h_{j}:\left[m_{j}\left(t_{0}\right), \infty\right) \longrightarrow \mathbb{R}, j=1, \ldots, N$ be arbitrary continuous functions. Suppose that condition (1.6) is fulfilled and $\tau_{j}(\cdot)$ is twice differentiable for all $j=1, \ldots, N$. If $x(t)$ is a solution of equation (1.8) and hence solution of (1.5) on an interval $\left[t_{0}, T\right)$ satisfying the initial condition $x(t)=\psi(t)$ on $\left[m\left(t_{0}\right), t_{0}\right]$ and $y\left(t_{0}\right)=\dot{x}\left(t_{0}\right)$, then $x(t)$ is the solution of the following integral equation

$$
\begin{aligned}
& \begin{aligned}
& x(t) \\
= & {\left[x\left(t_{0}\right)-\sum_{j=1}^{N} \int_{t_{0}-\tau_{j}\left(t_{0}\right)}^{t_{0}} h_{j}(v) x(v) d v\right] e^{-\int_{t_{0}}^{t} H(v) d v} }
\end{aligned} \\
& +\left[\dot{x}\left(t_{0}\right)+\sum_{j=1}^{N}\left(\omega_{j}\left(t_{0}\right) x\left(t_{0}-\tau_{j}\left(t_{0}\right)\right)-\int_{t_{0}-\tau_{j}\left(t_{0}\right)}^{t_{0}} C_{j}\left(t_{0}, v\right) g_{j}(v, x(v)) d v\right)\right] \\
& \times \int_{t_{0}}^{t} e^{-\int_{u}^{t} H(v) d v} e^{-\int_{t_{0}}^{u} A(v) d v} d u+\sum_{j=1}^{N} \int_{t-\tau_{j}(t)}^{t} h_{j}(v) x(v) d v \\
& -\sum_{j=1}^{N} \int_{t_{0}}^{t} e^{-\int_{u}^{t} H(v) d v} H(u) \int_{u-\tau_{j}(u)}^{u} h_{j}(v) x(v) d v d u \\
& +\sum_{j=1}^{N} \int_{t_{0}}^{t} e^{-\int_{u}^{t} H(v) d v}\left[\left(1-\tau_{j}^{\prime}(u)\right) h_{j}\left(u-\tau_{j}(u)\right)\right. \\
& \left.-\frac{b_{j}(u)}{1-\tau_{j}^{\prime}(u)}\right] x\left(u-\tau_{j}(u)\right) d u \\
& +\sum_{j=1}^{N} \int_{t_{0}}^{t} e^{-\int_{u}^{t} H(v) d v} \int_{u-\tau_{j}(u)}^{u} C_{j}(u, v) g_{j}(v, x(v)) d v d u \\
& -\sum_{j=1}^{N} \int_{t_{0}}^{t} e^{-\int_{u}^{t} H(v) d v} \int_{t_{0}}^{u} A(s) e^{-\int_{s}^{u} A(v) d v} \int_{s-\tau_{j}(s)}^{s} C_{j}(s, v) g_{j}(v, x(v)) d v d s d u \\
& +\sum_{j=1}^{N} \int_{t_{0}}^{t} e^{-\int_{u}^{t} H(v) d v} \int_{t_{0}}^{u} e^{-\int_{s}^{u} A(v) d v} C_{j}\left(s, s-\tau_{j}(s)\right)\left(1-\tau_{j}^{\prime}(s)\right) \\
& \times g_{j}\left(s-\tau_{j}(s), x\left(s-\tau_{j}(s)\right)\right) d s d u \\
& (3.9)+\sum_{j=1}^{N} \int_{t_{0}}^{t} e^{-\int_{u}^{t} H(v) d v} \int_{t_{0}}^{u} e^{-\int_{s}^{u} A(v) d v} r_{j}(s) x\left(s-\tau_{j}(s)\right) d s d u,
\end{aligned}
$$


on $\left[t_{0}, T\right)$, where $r_{j}(\cdot)$ and $H$ are respectively given by

$$
\begin{gathered}
r_{j}(t)=\left[\left(b_{j}(t) A(t)+b_{j}^{\prime}(t)\right)\left(1-\tau_{j}^{\prime}(t)\right)+b_{j}(t) \tau_{j}^{\prime \prime}(t)\right] /\left(1-\tau_{j}^{\prime}(t)\right)^{2} \\
H(t):=\sum_{j=1}^{N} h_{j}(t)
\end{gathered}
$$

for $t \in\left[m\left(t_{0}\right), \infty\right)$ and $m\left(t_{0}\right):=\inf _{1 \leq j \leq N} m_{j}\left(t_{0}\right)$.

Conversely, if a continuous function $x(\cdot)$ is equal to $\psi(\cdot)$ for $t \in\left[m\left(t_{0}\right), t_{0}\right]$ and is the solution of above integral equation on an interval $\left[t_{0}, T_{1}\right]$, then $x(\cdot)$ is a solution of (1.8) on $\left[t_{0}, T_{1}\right]$.

Proof. By Lemma (3.2), equation (3.1) can be written as

$\dot{x}(t)$

$$
\begin{aligned}
= & B(t)+\sum_{j=1}^{N} \int_{t_{0}}^{t} e^{-\int_{s}^{t} A(v) d v} \frac{d}{d s} \int_{s-\tau_{j}(s)}^{s} C_{j}(s, v) g_{j}(v, x(v)) d v d s \\
& +\sum_{j=1}^{N} \int_{t_{0}}^{t} e^{-\int_{s}^{t} A(v) d v}\left[C_{j}\left(s, s-\tau_{j}(s)\right)\left(1-\tau_{j}^{\prime}(s)\right) g_{j}\left(s-\tau_{j}(s), x\left(s-\tau_{j}(s)\right)\right)\right] d s \\
(3.12)- & \sum_{j=1}^{N} \int_{t_{0}}^{t} e^{-\int_{s}^{t} A(v) d v} \omega_{j}(s) \frac{d}{d s} x\left(s-\tau_{j}(s)\right) d s .
\end{aligned}
$$

Multiplying both sides of the above equation by $e^{\int_{t_{0}}^{t} H(v) d v}$ and integrating with respect to $u$ from $t_{0}$ to $t$, we obtain

$$
\begin{aligned}
x(t)= & x\left(t_{0}\right) e^{-\int_{t_{0}}^{t} H(v) d v}+\int_{t_{0}}^{t} B(u) e^{-\int_{u}^{t} H(v) d v} d u \\
& +\sum_{j=1}^{N} \int_{t_{0}}^{t} e^{-\int_{u}^{t} H(v) d v} \frac{d}{d u} \int_{u-\tau_{j}(u)}^{u} h_{j}(v) x(v) d v d u \\
& +\sum_{j=1}^{N} \int_{t_{0}}^{t} e^{-\int_{u}^{t} H(v) d v}\left(1-\tau_{j}^{\prime}(u)\right) h_{j}\left(u-\tau_{j}(u)\right) x\left(u-\tau_{j}(u)\right) d u \\
& +\sum_{j=1}^{N} \int_{t_{0}}^{t} e^{-\int_{u}^{t} H(v) d v} \int_{t_{0}}^{u} e^{-\int_{s}^{u} A(v) d v} \frac{d}{d s} \int_{s-\tau_{j}(s)}^{s} C_{j}(s, v) g_{j}(v, x(v)) d v d s d u \\
& +\sum_{j=1}^{N} \int_{t_{0}}^{t} e^{-\int_{u}^{t} H(v) d v} \int_{t_{0}}^{u} e^{-\int_{s}^{u} A(v) d v} C_{j}\left(s, s-\tau_{j}(s)\right)\left(1-\tau_{j}^{\prime}(s)\right) \\
& \left.-\sum_{j=1}^{N} \int_{t_{0}}^{t} e^{-\int_{u}^{t} H(v) d v} \int_{t_{0}}^{u} e^{-\int_{s}^{u} A(v) d v} \omega_{j}(s) \frac{d}{d s} x\left(s-\tau_{j}(s)\right) d s\left(s-\tau_{j}(s)\right)\right) d s d u
\end{aligned}
$$


Performing an integration by parts and using definitions (1.9) and (3.2), we obtain

$$
\begin{aligned}
& \begin{aligned}
& x(t) \\
= & {\left[x\left(t_{0}\right)-\sum_{j=1}^{N} \int_{t_{0}-\tau_{j}\left(t_{0}\right)}^{t_{0}} h_{j}(v) x(v) d v\right] e^{-\int_{t_{0}}^{t} H(v) d v} }
\end{aligned} \\
& +\left[\dot{x}\left(t_{0}\right)+\sum_{j=1}^{N}\left(\omega_{j}\left(t_{0}\right) x\left(t_{0}-\tau_{j}\left(t_{0}\right)\right)-\int_{t_{0}-\tau_{j}\left(t_{0}\right)}^{t_{0}} C_{j}\left(t_{0}, v\right) g_{j}(v, x(v)) d v\right)\right] \\
& \times \int_{t_{0}}^{t} e^{-\int_{u}^{t} H(v) d v} e^{-\int_{t_{0}}^{u} A(v) d v} d u \\
& +\sum_{j=1}^{N} \int_{t-\tau_{j}(t)}^{t} h_{j}(v) x(v) d v-\sum_{j=1}^{N} \int_{t_{0}}^{t} e^{-\int_{u}^{t} H(v) d v} H(u) \int_{u-\tau_{j}(u)}^{u} h_{j}(v) x(v) d v d u \\
& +\sum_{j=1}^{N} \int_{t_{0}}^{t} e^{-\int_{u}^{t} H(v) d v}\left[\left(1-\tau_{j}^{\prime}(u)\right) h_{j}\left(u-\tau_{j}(u)\right)-\frac{b(u)}{1-\tau_{j}^{\prime}(u)}\right] x\left(u-\tau_{j}(u)\right) d u \\
& +\sum_{j=1}^{N} \int_{t_{0}}^{t} e^{-\int_{u}^{t} H(v) d v} \int_{u-\tau_{j}(u)}^{u} C_{j}(u, v) g_{j}(v, x(v)) d v d u \\
& -\sum_{j=1}^{N} \int_{t_{0}}^{t} e^{-\int_{u}^{t} H(v) d v} \int_{t_{0}}^{u} A(s) e^{-\int_{s}^{u} A(v) d v} \int_{s-\tau_{j}(s)}^{s} C_{j}(s, v) g_{j}(v, x(v)) d v d s d u \\
& +\sum_{j=1}^{N} \int_{t_{0}}^{t} e^{-\int_{u}^{t} H(v) d v} \int_{t_{0}}^{u} e^{-\int_{s}^{u} A(v) d v} C_{j}\left(s, s-\tau_{j}(s)\right)\left(1-\tau_{j}^{\prime}(s)\right) \\
& \times g_{j}\left(s-\tau_{j}(s), x\left(s-\tau_{j}(s)\right)\right) d s d u \\
& +\sum_{j=1}^{N} \int_{t_{0}}^{t} e^{-\int_{u}^{t} H(v) d v} \int_{t_{0}}^{u} e^{-\int_{s}^{u} A(v) d v} r_{j}(t) x\left(s-\tau_{j}(s)\right) d s d u
\end{aligned}
$$

where $r_{j}(t)$ is defined in (3.10). This leads exactly to (3.9).

Conversely, suppose that a continuous function $x(\cdot)$ is equal to $\psi(\cdot)$ on $\left[m\left(t_{0}\right), t_{0}\right]$ and satisfies $(3.9)$ on an interval $\left[t_{0}, T_{1}\right)$. Then it is twice differentiable on $\left[t_{0}, T_{1}\right)$. Differentiating (3.9) with the aid of Leibniz's rule, we obtain (1.5).

Next, we will define a mapping directly from (3.9). Remember that, by Lemma 3.3 a fixed point of that map will be a solution of equation (1.5). To obtain stability of the zero solution of (1.5), we need the mapping defined by (3.9) to map bounded functions into bounded functions. For that, we let $(\mathcal{C},\|\cdot\|)$ to be the Banach space of real-valued bounded continuous functions on $\left[m\left(t_{0}\right), \infty\right)$ with the supremum norm $\|\cdot\|$, that is for $\varphi \in \mathcal{C}$

$$
\|\varphi\|:=\sup \left\{|\varphi(t)|, t \in\left[m\left(t_{0}\right), \infty\right)\right\}
$$


Our investigations will be carried out on the complete metric space $(\mathcal{C}, \rho)$, where $\rho$ is the uniform metric. That is, for $\varphi, \phi \in \mathcal{C}$ we set $\rho(\varphi, \phi)=\|\varphi-\phi\|$.

Let $\psi \in \mathcal{C}\left(\left[m\left(t_{0}\right), t_{0}\right], \mathbb{R}\right)$ be fixed and define

$$
S_{\psi}:=\left\{\varphi:\left[m\left(t_{0}\right), \infty\right) \rightarrow \mathbb{R} \mid \varphi \in \mathcal{C}, \varphi(t)=\psi(t) \text { for } t \in\left[m\left(t_{0}\right), t_{0}\right]\right\} .
$$

Being closed in $\mathcal{C},\left(S_{\psi}, \rho\right)$ is itself complete. There is no confusion if we use the norm $\|\cdot\|$ on $\left[m\left(t_{0}\right), t_{0}\right]$ or on $\left[m\left(t_{0}\right), \infty\right)$.

Below we want to force the mapping suggested by (3.9) and explicitly defined in the next lemma to map $S_{\psi}$ into itself. For that reason we assume that the followings conditions hold.

i.

$$
\liminf _{t \rightarrow \infty} \int_{t_{0}}^{t} H(s) d s>-\infty .
$$

ii. There exists some functions $R_{j}(\cdot) \in \mathcal{C}\left(\mathbb{R}, \mathbb{R}^{+}\right)$such that, for $x_{1}, x_{2} \in \mathbb{R}$ (3.14) $\left|g_{j}\left(t, x_{1}\right)-g_{j}\left(t, x_{2}\right)\right| \leq R_{j}(t)\left|x_{1}-x_{2}\right|, j=1, \ldots, N$ for all $t \in \mathbb{R}$,

$$
g_{j}(t, 0)=0, j=1, \ldots, N \text { for } t \in \mathbb{R}^{+} .
$$

ii. For $t \geq t_{0}$, there is a constant $\alpha>0$ satisfying

$$
\begin{aligned}
& \sum_{j=1}^{N} \int_{t-\tau_{j}(t)}^{t}\left|h_{j}(v)\right| d v+\sum_{j=1}^{N} \int_{t_{0}}^{t} e^{-\int_{u}^{t} H(v) d v}|H(u)| \int_{u-\tau_{j}(u)}^{u}\left|h_{j}(v)\right| d v d u \\
& +\sum_{j=1}^{N} \int_{t_{0}}^{t} e^{-\int_{u}^{t} H(v) d v \mid}\left|\left(1-\tau_{j}^{\prime}(u)\right) h_{j}\left(u-\tau_{j}(u)\right)-\frac{b_{j}(u)}{1-\tau_{j}^{\prime}(u)}\right| d u \\
& +\sum_{j=1}^{N} \int_{t_{0}}^{t} e^{-\int_{u}^{t} H(v) d v} \int_{u-\tau_{j}(u)}^{u}\left|C_{j}(u, v)\right| R_{j}(v) d v d u \\
& +\sum_{j=1}^{N} \int_{t_{0}}^{t} e^{-\int_{u}^{t} H(v) d v} \int_{t_{0}}^{u} A(s) e^{-\int_{s}^{u} A(v) d v} \int_{s-\tau_{j}(s)}^{s}\left|C_{j}(s, v)\right| R_{j}(v) d v d s d u \\
& +\sum_{j=1}^{N} \int_{t_{0}}^{t} e^{-\int_{u}^{t} H(v) d v} \int_{t_{0}}^{u} e^{-\int_{s}^{u} A(v) d v}\left|C_{j}\left(s, s-\tau_{j}(s)\right)\left(1-\tau_{j}^{\prime}(s)\right)\right| \\
& \times R_{j}\left(s-\tau_{j}(s)\right) d s d u \\
& +\sum_{j=1}^{N} \int_{t_{0}}^{t} e^{-\int_{u}^{t} H(v) d v} \int_{t_{0}}^{u} e^{-\int_{s}^{u} A(v) d v}\left|r_{j}(s)\right| d s d u \\
& \leq \alpha
\end{aligned}
$$

iii. There exist constants $a_{0}>0, \gamma>0, Q_{0}>0$ and a continuous function $A_{1} \in \mathcal{C}\left(\mathbb{R}^{+}, \mathbb{R}^{+}\right)$such that, for $t \geq t_{0}$

$$
f(t, x, y) \geq A_{1}(t) \geq 0 \text { for all } x, y \in \mathbb{R},
$$


and for each $t \geq u \geq Q_{0}$ we have

$$
\int_{u}^{t} H(v) d v+\int_{t_{0}}^{u} A_{1}(v) d v \geq a_{0} u^{\gamma}+b, b \in \mathbb{R} .
$$

ii. There exists a constant $\beta>0$ that satisfies the following inequality for $t \geq t_{0}$ (3.19)

$\sum_{j=1}^{N} \frac{\left|b_{j}(t)\right|}{\left|1-\tau_{j}^{\prime}(t)\right|}+\sum_{j=1}^{N} \int_{t_{0}}^{t} e^{-\int_{s}^{t} A(u) d u}\left(\int_{s-\tau_{j}(s)}^{s}\left|a_{j}(s, v)\right| R_{j}(v) d v+\left|r_{j}(s)\right|\right) d s \leq \beta$.

Lemma 3.4. Define the mapping $P$ on $S_{\psi}$ as follows, for $\varphi \in S_{\psi}$,

$$
(P \varphi)(t)=\psi(t) \text { if } t \in\left[m\left(t_{0}\right), t_{0}\right]
$$

while for $t>t_{0}$

$$
\begin{aligned}
& (P \varphi)(t)=\left[\psi\left(t_{0}\right)-\sum_{j=1}^{N} \int_{t_{0}-\tau_{j}\left(t_{0}\right)}^{t_{0}} h_{j}(v) \psi(v) d v\right] e^{-\int_{t_{0}}^{t} H(v) d v} \\
& +\left[\dot{x}\left(t_{0}\right)+\sum_{j=1}^{N}\left(\frac{b_{j}\left(t_{0}\right)}{1-\tau_{j}^{\prime}\left(t_{0}\right)} \psi\left(t_{0}-\tau_{j}\left(t_{0}\right)\right)\right.\right. \\
& \left.\left.-\int_{t_{0}-\tau_{j}\left(t_{0}\right)}^{t_{0}} C_{j}\left(t_{0}, v\right) g_{j}(v, \psi(v)) d v\right)\right] \\
& \times \int_{t_{0}}^{t} e^{-\int_{u}^{t} H(v) d v} e^{-\int_{t_{0}}^{u} A(v) d v} d u+\sum_{j=1}^{N} \int_{t-\tau_{j}(t)}^{t} h_{j}(v) \varphi(v) d v \\
& -\sum_{j=1}^{N} \int_{t_{0}}^{t} e^{-\int_{u}^{t} H(v) d v} H(u) \int_{u-\tau_{j}(u)}^{u} h_{j}(v) \varphi(v) d v d u \\
& +\sum_{j=1}^{N} \int_{t_{0}}^{t} e^{-\int_{u}^{t} H(v) d v}\left[\left(1-\tau_{j}^{\prime}(u)\right) h_{j}\left(u-\tau_{j}(u)\right)-\frac{b_{j}(u)}{1-\tau_{j}^{\prime}(u)}\right] \varphi\left(u-\tau_{j}(u)\right) d u \\
& +\sum_{j=1}^{N} \int_{t_{0}}^{t} e^{-\int_{u}^{t} H(v) d v} \int_{u-\tau_{j}(u)}^{u} C_{j}(u, v) g_{j}(v, \varphi(v)) d v d u \\
& -\sum_{j=1}^{N} \int_{t_{0}}^{t} e^{-\int_{u}^{t} H(v) d v} \int_{t_{0}}^{u} A(s) e^{-\int_{s}^{u} A(v) d v} \int_{s-\tau_{j}(s)}^{s} C_{j}(s, v) g_{j}(v, \varphi(v)) d v d s d u \\
& +\sum_{j=1}^{N} \int_{t_{0}}^{t} e^{-\int_{u}^{t} H(v) d v} \int_{t_{0}}^{u} e^{-\int_{s}^{u} A(v) d v} C_{j}\left(s, s-\tau_{j}(s)\right)\left(1-\tau_{j}^{\prime}(s)\right) \\
& \times g_{j}\left(s-\tau_{j}(s), x\left(s-\tau_{j}(s)\right)\right) d s d u \\
& +\sum_{j=1}^{N} \int_{t_{0}}^{t} e^{-\int_{u}^{t} H(v) d v} \int_{t_{0}}^{u} e^{-\int_{s}^{u} A(v) d v} r_{j}(s) \varphi\left(s-\tau_{j}(s)\right) d s d u
\end{aligned}
$$


where $r_{j}(\cdot)$ is the expression (3.10). Suppose that the conditions (3.13), (3.14), (3.15)), (3.16), (3.17) and (3.18) hold true. Then $P: S_{\psi} \rightarrow S_{\psi}$.

Proof. First, due to condition (3.13) one can define

$$
M=\sup _{t \geq t_{0}}\left\{e^{-\int_{t_{0}}^{t} H(v) d v}\right\} .
$$

Obviously, if $\varphi$ is continuous then $P \varphi$ and agrees with $\psi$ on $\left[m\left(t_{0}\right), t_{0}\right]$ due to the definition of $P$. For $t>t_{0}$, note that from (3.13), (3.16), (3.14) and (3.15) it follows

$$
\begin{aligned}
|P \varphi(t)|= & \|\psi\|\left[1+\sum_{j=1}^{N} \int_{t_{0}-\tau_{j}\left(t_{0}\right)}^{t_{0}}\left|h_{j}(v)\right| d v\right] M \\
& +\left[\left|\dot{x}\left(t_{0}\right)\right|+\|\psi\| \sum_{j=1}^{N}\left(\left|\frac{b_{j}\left(t_{0}\right)}{1-\tau_{j}^{\prime}\left(t_{0}\right)}\right|+\int_{t_{0}-\tau_{j}\left(t_{0}\right)}^{t_{0}}\left|C_{j}\left(t_{0}, v\right)\right| R_{j}(t) d v\right)\right] \\
& \times \int_{t_{0}}^{t} e^{-\int_{u}^{t} H(v) d v} e^{-\int_{t_{0}}^{u} A(v) d v} d u+\alpha\|\varphi\| .
\end{aligned}
$$

To prove that $P: S_{\psi} \rightarrow S_{\psi}$ it is necessary to show that the term

$$
\int_{t_{0}}^{t} e^{-\int_{u}^{t} H(v) d v} e^{-\int_{t_{0}}^{u} A(v) d v} d u
$$

is bounded. To do that, remember that 3.17 implies that $A(t) \geq A_{1}(t) \geq 0$ for $t \geq t_{0}$, so

$$
\int_{t_{0}}^{t} e^{-\int_{u}^{t} H(v) d v} e^{-\int_{t_{0}}^{u} A(v) d v} d u \leq \int_{t_{0}}^{t} e^{-\int_{u}^{t} H(v) d v} e^{-\int_{t_{0}}^{u} A_{1}(v) d v} d u .
$$

We decompose the last integral term in the following manner

$$
\begin{aligned}
\int_{t_{0}}^{t} e^{-\int_{u}^{t} H(v) d v} e^{-\int_{t_{0}}^{u} A_{1}(v) d v} d u= & \int_{t_{0}}^{J} e^{-\int_{u}^{t} H(v) d v} e^{-\int_{t_{0}}^{u} A_{1}(v) d v} d u \\
& +\int_{J}^{t} e^{-\int_{u}^{t} H(v) d v} e^{-\int_{t_{0}}^{u} A_{1}(v) d v} d u
\end{aligned}
$$

for some $J \geq Q_{0}$. The first term on the right hand side of (3.22) is obviously bounded. For the second term on the right hand side of (3.22), we use (3.18) to have

$$
\int_{J}^{t} e^{-\int_{u}^{t} H(v) d v} e^{-\int_{t_{0}}^{u} A_{1}(v) d v} d u \leq e^{-b} \int_{J}^{t} e^{-a_{0} u^{\gamma}} d u .
$$

Now, we define

$$
F(J):=\int_{J}^{\infty} e^{-a_{0} u^{\gamma}} d u
$$


Performing the change of variables $u=\theta^{\frac{1}{\gamma}}$, we obtain

$$
F(J)=\frac{1}{\gamma} \int_{J^{\gamma}}^{\infty} e^{-a_{0} \theta} \theta^{\frac{1}{\gamma}-1} d \theta \leq \frac{1}{\gamma} \int_{0}^{\infty} e^{-a_{0} \theta} \theta^{\frac{1}{\gamma}-1} d \theta=\frac{\Gamma(1 / \gamma)}{\gamma a_{0}^{1 / \gamma}} .
$$

Then $F(J)$ is bounded for $\gamma>0$. Consequently, $|P \varphi(t)|<+\infty$ and thus $P \varphi \in$ $S_{\psi}$.

Basing on Lemma (3.3) and Lemma (3.4) we built an existence and uniqueness result. Under the conditions of the next theorem, we prove that for a given continuous function $\psi:\left[m\left(t_{0}\right), t_{0}\right] \longrightarrow \mathbb{R}$ there exists a unique continuous function $x$ which is solution of $(1.5)$ on $\left[m\left(t_{0}\right), \infty\right)$ and coincides with $\psi$ on $\left[m\left(t_{0}\right), t_{0}\right]$. We also prove that the zero solution of (1.5) have the property of Definition 2.1.

Theorem 3.1. Suppose the condition (3.19) and all hypotheses of Lemma (3.4) hold with $\alpha \in(0,1)$ in (3.16). Then, for each initial continuous function $\psi$ : $\left[m\left(t_{0}\right), t_{0}\right] \longrightarrow \mathbb{R}$, there is a unique continuous function with $x(t)=\psi(t)$ on $\left[m\left(t_{0}\right), t_{0}\right]$ that satisfies (1.5) on $\left[t_{0}, \infty\right)$. Moreover, $x(\cdot)$ is bounded on $\left[m\left(t_{0}\right), \infty\right)$. Furthermore, the zero solution of (1.5) is stable at $t=t_{0}$.

Proof. Consider $S_{\psi}$ the space defined by the initial continuous function $\psi:\left[m\left(t_{0}\right), t_{0}\right] \rightarrow$ $\mathbb{R}$. By Lemma 3.4 we know that $P: S_{\psi} \rightarrow S_{\psi}$. In fact, $P$ is a contraction with constant $\alpha<1$ too. To see this, let $\varphi, \phi \in S_{\psi}$. Making use of condition (3.16) we obtain

$$
\begin{aligned}
& \|P \varphi-P \phi\| \\
& \leq\left[\sum_{j=1}^{N} \int_{t-\tau_{j}(t)}^{t}\left|h_{j}(v)\right| d v+\sum_{j=1}^{N} \int_{t_{0}}^{t} e^{-\int_{u}^{t} H(v) d v}|H(u)| \int_{u-\tau_{j}(u)}^{u}\left|h_{j}(v)\right| d v d u\right. \\
& +\sum_{j=1}^{N} \int_{t_{0}}^{t} e^{-\int_{u}^{t} H(v) d v}\left|\left(1-\tau_{j}^{\prime}(u)\right) h_{j}\left(u-\tau_{j}(u)\right)-\frac{b_{j}(u)}{1-\tau_{j}^{\prime}(u)}\right| d u \\
& +\sum_{j=1}^{N} \int_{t_{0}}^{t} e^{-\int_{u}^{t} H(v) d v} \int_{u-\tau_{j}(u)}^{u}\left|C_{j}(u, v)\right| R_{j}(v) d v d u \\
& +\sum_{j=1}^{N} \int_{t_{0}}^{t} e^{-\int_{u}^{t} H(v) d v} \int_{t_{0}}^{u} A(s) e^{-\int_{s}^{u} A(v) d v} \int_{s-\tau_{j}(s)}^{s}\left|C_{j}(s, v)\right| R_{j}(v) d v d s d u \\
& +\sum_{j=1}^{N} \int_{t_{0}}^{t} e^{-\int_{u}^{t} H(v) d v} \int_{t_{0}}^{u} e^{-\int_{s}^{u} A(v) d v}\left|C_{j}\left(s, s-\tau_{j}(s)\right)\left(1-\tau_{j}^{\prime}(s)\right)\right| \\
& \times R_{j}\left(s-\tau_{j}(s)\right) d s d u \\
& \left.+\sum_{j=1}^{N} \int_{t_{0}}^{t} e^{-\int_{u}^{t} H(v) d v} \int_{t_{0}}^{u} e^{-\int_{s}^{u} A(v) d v}\left|r_{j}(s)\right| d s d u\right]\|\varphi-\phi\| \\
& \leq \alpha\|\varphi-\phi\|,
\end{aligned}
$$


for $t>t_{0}$. Trivially, this inequality also holds on $\left[m\left(t_{0}\right), t_{0}\right]$. Therefore, $P$ is a contraction mapping on the complete metric space $\left(S_{\psi}, \rho\right)$ since we have supposed $\alpha<1$. By the contraction mapping principle, $P$ possesses a unique fixed point $x$ in $S_{\psi}$ which is bounded continuous function. Due to Lemma 3.3, this is a solution of $(1.8)$ and hence a solution of $(1.5)$ on $\left[m\left(t_{0}\right), \infty\right)$. It follows that $x$ is the only bounded function satisfying (1.5) on $\left[m\left(t_{0}\right), \infty\right)$ and the initial function.

It remains to show that the zero solution of (1.5) is stable. Toward this, let first

$$
L:=\sup _{t \geq t_{0}} \int_{t_{0}}^{t} e^{-\int_{u}^{t} H(v) d v} e^{-\int_{t_{0}}^{u} A_{1}(v) d v} d u .
$$

Let $\epsilon>0$ be given. Choose $\left|\dot{x}\left(t_{0}\right)\right|$ and $\psi:\left[m\left(t_{0}\right), t_{0}\right] \longrightarrow \mathbb{R}$ satisfying $\|\psi\|<\delta$ $(\delta \leq \varepsilon)$, with $\delta$ such that

$$
\begin{aligned}
\delta & {\left[1+\sum_{j=1}^{N} \int_{t_{0}-\tau_{j}\left(t_{0}\right)}^{t_{0}}\left|h_{j}(v)\right| d v\right] M } \\
+ & {\left[\left|\dot{x}\left(t_{0}\right)\right|+\delta \sum_{j=1}^{N}\left(\left|\frac{b_{j}\left(t_{0}\right)}{1-\tau_{j}^{\prime}\left(t_{0}\right)}\right|+\int_{t_{0}-\tau_{j}\left(t_{0}\right)}^{t_{0}}\left|C_{j}\left(t_{0}, v\right)\right| R_{j}(t) d v\right)\right] L } \\
(3.27) \leq & (1-\alpha) \epsilon .
\end{aligned}
$$

If $(x(t), y(t))$ is a solution of (1.8) with $y=\dot{x}$ on $\left[t_{0}, \infty\right)$ and, $y\left(t_{0}\right)=\dot{x}\left(t_{0}\right)$ then, $x(\cdot)=(P x)(\cdot)$ defined in (3.20). Notice that with such a $\delta,|x(s)|=|\psi(s)|<\epsilon$ on $\left[m\left(t_{0}\right), t_{0}\right]$. We claim that $|x(t)|<\epsilon$ for all $t \geq t_{0}$. If $x$ is a solution with initial function $\psi$ then, as consequence of (3.20), we have

$$
\begin{aligned}
\leq & {[\delta(t) \mid} \\
\leq & {\left[\delta \sum_{j=1}^{N} \int_{t_{0}-\tau_{j}\left(t_{0}\right)}^{t_{0}}\left|h_{j}(v)\right| d v\right] M } \\
& +\left[\left|\dot{x}\left(t_{0}\right)\right|+\delta \sum_{j=1}^{N} \frac{\left|b_{j}\left(t_{0}\right)\right|}{\left|1-\tau_{j}^{\prime}\left(t_{0}\right)\right|}+\delta \int_{t_{0}-\tau_{j}\left(t_{0}\right)}^{t_{0}}\left|C_{j}\left(t_{0}, v\right)\right| R_{j}(v) d v\right] L \\
& +\varepsilon \alpha
\end{aligned}
$$$$
(3.28) \leq(1-\alpha) \varepsilon+\varepsilon \alpha \leq \varepsilon .
$$

Now, recalling (3.1) of Lemma 3.1, we have

$$
\begin{aligned}
\dot{x}(t)= & \dot{x}\left(t_{0}\right) e^{-\int_{t_{0}}^{t} A(v) d v} \\
& -\sum_{j=1}^{N} \int_{t_{0}}^{t} e^{-\int_{s}^{t} A(v) d v} \int_{s-\tau_{j}(s)}^{s} a_{j}(s, v) g_{j}(v, x(v)) d v d s \\
& -\sum_{j=1}^{N} \int_{t_{0}}^{t} e^{-\int_{s}^{t} A(v) d v} \omega_{j}(s) \frac{d}{d s} x\left(s-\tau_{j}(s)\right) d s .
\end{aligned}
$$


Integrating the last term on right hand side by parts we obtain

$$
\begin{aligned}
\dot{x}(t)= & e^{-\int_{t_{0}}^{t} A(v) d v}\left(\dot{x}\left(t_{0}\right)+\sum_{j=1}^{N} \frac{b_{j}\left(t_{0}\right)}{1-\tau_{j}^{\prime}\left(t_{0}\right)} x\left(t_{0}-\tau_{j}\left(t_{0}\right)\right)\right) \\
& -\sum_{j=1}^{N} \frac{b_{j}(t)}{1-\tau_{j}^{\prime}(t)} x\left(t-\tau_{j}(t)\right) \\
& +\int_{t_{0}}^{t} e^{-\int_{s}^{t} A(v) d v} \sum_{j=1}^{N}\left(r_{j}(s) x\left(s-\tau_{j}(s)\right)-\int_{s-\tau_{j}(s)}^{s} a_{j}(s, v) g_{j}(v, x(v)) d v\right) d s .
\end{aligned}
$$

By conditions (3.27) and (3.19) we get the estimation

$$
\begin{aligned}
& |\dot{x}(t)| \leq\left|\dot{x}\left(t_{0}\right)\right|+\delta \sum_{j=1}^{N} \frac{\left|b_{j}\left(t_{0}\right)\right|}{\left|1-\tau_{j}^{\prime}\left(t_{0}\right)\right|}+\varepsilon \sum_{j=1}^{N}\left[\frac{\left|b_{j}(t)\right|}{\left|1-\tau_{j}^{\prime}(t)\right|}\right. \\
& \left.+\int_{t_{0}}^{t} e^{-\int_{s}^{t} A(u) d u}\left(\left|r_{j}(s)\right|+\int_{s-\tau_{j}(s)}^{s}\left|a_{j}(s, v)\right| R_{j}(v) d v\right) d s\right] \\
& \leq \frac{(1-\alpha) \varepsilon}{L}+\varepsilon \beta \leq \varepsilon\left(\frac{1}{L}+\beta\right) \text {. }
\end{aligned}
$$

Therefore, the zero solution is stable at $t=t_{0}$.

Theorem 3.2. Under the hypotheses of Theorem3.1 the zero solution of (1.5) is asymptotically stable if and only if

$$
\int_{t_{0}}^{t} H(s) d s \longrightarrow \infty, \text { as } t \longrightarrow \infty \text {. }
$$

Proof. First, suppose that (3.29) holds. We wish the solutions of (1.5) to tend to zero whenever condition (3.29) holds. For this, we will modify $S_{\psi}$ in order to receipt functions that tends to zero as $t \longrightarrow \infty$. So, we let

$$
\begin{aligned}
& S_{\psi}^{0} \quad: \quad=\left\{\varphi \in\left[m\left(t_{0}\right), \infty\right) \rightarrow \mathbb{R} \mid \varphi \in \mathcal{C},\right. \\
&\left.\varphi(t)=\psi(t) \text { for } t \in\left[m\left(t_{0}\right), t_{0}\right] \text { and } \varphi(t) \rightarrow 0 \text { as } t \rightarrow \infty\right\} .
\end{aligned}
$$

Since $S_{\psi}^{0}$ is closed in $S_{\psi}$ and $\left(S_{\psi}, \rho\right)$ is complete, then the metric space $\left(S_{\psi}^{0}, \rho\right)$ is also complete. We begin by proving that $P \varphi(t) \rightarrow 0$ as $t \rightarrow \infty$ for $\varphi \in S_{\psi}^{0}$. To this end, denote the nine terms on the right hand side of $(3.20)$ by $I_{1}, I_{2}, \ldots, I_{9}$, respectively and let $\varphi \in S_{\psi}^{0}$ be fixed. Since $\int_{0}^{t} H(s) d s \longrightarrow \infty$, as $t \longrightarrow \infty$, by condition (3.29), we see obviously that the first term $I_{1}$ of (3.20) tends to zero as $t \longrightarrow \infty$. For a given $\epsilon>0$, choose $T_{0}>0$ large enough so that $t-\tau_{j}(t) \geq T_{0}$ for $j=\overline{1, N}$ implies $|\varphi(s)|<\epsilon$ if $s \geq t-\tau_{j}(t)$. Therefore, the third term $I_{3}$ in (3.20) 
satisfies

$$
\begin{aligned}
\left|I_{3}\right| & \leq \sum_{j=1}^{N} \int_{t-\tau_{j}(t)}^{t}|\varphi(v)|\left|h_{j}(v)\right| d v \leq \varepsilon \sum_{j=1}^{N} \int_{t-\tau_{j}(t)}^{t}\left|h_{j}(v)\right| d v \\
& \leq \alpha \epsilon<\epsilon .
\end{aligned}
$$

Thus, $I_{3} \longrightarrow 0$ as $t \longrightarrow \infty$. We check that $I_{2} \longrightarrow 0$ as $t \longrightarrow \infty$. So we have to prove that the two right hand side terms of the decomposition expression (3.22) go to zero at infinity. But the first term of that decomposition is as

$$
\int_{t_{0}}^{J} e^{-\int_{u}^{t} H(v) d v} e^{-\int_{t_{0}}^{u} A_{1}(v) d v} d u=e^{-\int_{J}^{t} H(v) d v} \int_{t_{0}}^{J} e^{-\int_{u}^{J} H(v) d v} e^{-\int_{t_{0}}^{u} A_{1}(v) d v} d u,
$$

which tends to 0 as $t \longrightarrow \infty$ by condition (3.29). Nevertheless, the second term of on the right had side of (3.22) needs some more details for its convergence to zero. To overcome the difficulties, remember that from (3.18) we have obtained (3.24). Upon replacing of $u$ by $J \theta$ in (3.24) we get

$$
F(J)=J \int_{1}^{\infty} e^{-\left(a_{0} J^{\gamma}\right) \theta^{\gamma}} d \theta
$$

The function $G(\lambda):=\int_{1}^{\infty} e^{-\lambda \theta^{\gamma}} d \theta$ satisfies the conditions of Theorem 2.1 where (3.31) $\lambda=a_{0} J^{\gamma}, \quad \alpha=1, \quad \varphi(\theta)=\theta^{\gamma}, \quad f \equiv 1 \quad \varphi^{\prime}(\alpha)=\gamma \alpha^{\gamma-1}=\gamma \quad f(\alpha)=1$.

It follows that

$$
G(\lambda) \sim \frac{f(\alpha)}{\varphi^{\prime}(\alpha)} \frac{1}{\lambda} e^{-\lambda \varphi(\alpha)}=\frac{1}{\gamma} \frac{1}{\lambda} e^{-\lambda}, \quad(\lambda \longrightarrow+\infty) .
$$

Thus we can write

$$
F(J) \sim \frac{1}{\gamma a_{0}} J^{1-\gamma} e^{-a_{0} J^{\gamma}}, \quad(J \longrightarrow+\infty) .
$$

It is enough to make $z=a_{0} J^{\gamma}$ and a straightforward computation gives

$$
\frac{1}{\gamma a_{0}} J^{1-\gamma} e^{-a_{0} J^{\gamma}}=\frac{1}{\gamma a_{0}^{1 / \gamma}} z^{\frac{1}{\gamma}-1} e^{-z} \leq \frac{1}{\gamma a_{0}^{1 / \gamma}} z^{m} e^{-z} \longrightarrow 0 \text { as } z \longrightarrow \infty .
$$

where $m:=[1 / \gamma]+1$. Thus, for every $\epsilon>0$ we can find a $J^{*} \gg Q_{0}$ large enough such that for every $J \geq J^{*}$

$$
\frac{e^{-b}}{\gamma a_{0}} J^{1-\gamma} e^{-a_{0} J^{\gamma}} \leq \epsilon .
$$

Clearly, the expansion (3.22) is valid if $J$ is replaced by $J^{*}$. So, the last term tends towards zero when $t \longrightarrow \infty$. Hence the second term $I_{2}$ in (3.20) tends to zero as $t \longrightarrow \infty$. Now consider $I=\left|I_{4}\right|+\ldots+\left|I_{9}\right|$. To simplify our expressions, we define 


$$
\begin{aligned}
V(u) \quad: \quad & =\sum_{j=1}^{N} \int_{u-\tau_{j}(u)}^{u}\left[\left|h_{j}(v) H(u)\right|+\left|C_{j}(u, v)\right| R_{j}(v)\right] d v \\
& +\sum_{j=1}^{N}\left|\left(1-\tau_{j}^{\prime}(u)\right) h_{j}\left(u-\tau_{j}(u)\right)-\frac{b_{j}(u)}{1-\tau_{j}^{\prime}(u)}\right| \\
& +\sum_{j=1}^{N} \int_{t_{0}}^{u} e^{-\int_{s}^{u} A(v) d v}\left|C_{j}\left(s, s-\tau_{j}(s)\right)\left(1-\tau_{j}^{\prime}(s)\right)\right| R_{j}\left(s-\tau_{j}(s)\right) d s \\
& +\sum_{j=1}^{N} \int_{t_{0}}^{u} e^{-\int_{s}^{u} A(v) d v}\left(A(s) \int_{s-\tau_{j}(s)}^{s}\left|C_{j}(s, v)\right| R_{j}(v) d v+\left|r_{j}(s)\right|\right) d s .
\end{aligned}
$$

So, for the given $\epsilon>0$, there exists a $T^{*}>t_{0}$ such that $s \geq T^{*}$ implies $\left|\varphi\left(s-\tau_{j}(s)\right)\right|<$ $\epsilon$ for $j=\overline{1, N}$. It is clear that $|\varphi(s)|<\varepsilon$ ( because $s>s-\tau_{j}(s), j=\overline{1, N}$ ). Thus, for $t \geq T^{*}$, by making use conditions (3.14) and (3.15) the term $I$ satisfies

$$
\begin{aligned}
I \leq & \epsilon \sum_{j=1}^{N} \int_{t-\tau_{j}(t)}^{t}\left|h_{j}(v)\right| d v+\varepsilon \int_{T^{*}}^{t} V(u) e^{-\int_{u}^{t} H(v) d v} d u \\
& +\sup _{\zeta \geq m\left(t_{0}\right)}|\varphi(\zeta)| \int_{t_{0}}^{T^{*}} V(u) e^{-\int_{u}^{t} H(v) d v} d u \\
\leq & 2 \alpha \epsilon+\sup _{\zeta \geq m\left(t_{0}\right)}|\varphi(\zeta)| \int_{t_{0}}^{T^{*}} V(u) e^{-\int_{u}^{t} H(v) d v} d u
\end{aligned}
$$

Also, the conditions (3.29) implies that, there exists $T^{* *}>T^{*}$ such that for $t \geq T^{* *}$ we have

$$
e^{-\int_{T^{* *}}^{t} H(v) d v} \sup _{\zeta \geq m\left(t_{0}\right)}|\varphi(\zeta)| \int_{T^{*}}^{t} V(u) e^{-\int_{u}^{T^{* *}} H(v) d v} d u \leq \epsilon
$$

So, $I \longrightarrow 0$ as $t \longrightarrow \infty$ and consequently, $(P \varphi)(t) \longrightarrow 0$ as $t \longrightarrow \infty$. Thus, $P$ maps $S_{\psi}^{0}$ into itself. Also, $P$ is still a contraction on $S_{\psi}^{0}$ with a unique fixed point $x$. By Lemma 3.3, $x$ is a solution of $(1.5)$ on $\left[t_{0}, \infty\right)$. We conclude that $x(t)$ is the only continuous solution of (1.5) agreeing with the initial function $\psi$. As $x \in S_{\psi}^{0}$, $x(t) \rightarrow 0$ as $t \rightarrow \infty$. Therefore, the zero solution is asymptotically stable, since it is stable by Theorem 3.1 and we have just concluded that $|x(t)|+|y(t)| \longrightarrow 0$ as $t \longrightarrow \infty$ if condition (3.29) holds.

Conversely, we shall prove that $\int_{t_{0}}^{\infty} H(v) d v=\infty$. Contrary to this, there exists a sequence $\left\{t_{n}\right\}_{n \geq 1}$ with $t_{n} \longrightarrow \infty$ as $n \longrightarrow \infty$ and such that $\int_{t_{0}}^{t_{n}} H(v) d v=l$ for a certain finite number $l \in \mathbb{R}^{+}$. By condition (3.13), we may also choose $\mu>0$ that satisfies the inequality, $-\mu \leq \int_{t_{0}}^{t_{n}} H(v) d v \leq \mu$, for all $n \geq 1$. For convenience of 
notation we set

$$
C_{0}:=\sum_{j=1}^{N}\left(\frac{\left|b_{j}\left(t_{0}\right)\right|}{\left|1-\tau_{j}^{\prime}\left(t_{0}\right)\right|}\left|\psi\left(t_{0}-\tau_{j}\left(t_{0}\right)\right)\right|+\int_{t_{0}-\tau_{j}\left(t_{0}\right)}^{t_{0}}\left|C_{j}\left(t_{0}, v\right)\right| R_{j}(v)|\psi(v)| d v\right) .
$$

Recalling (3.36), we define the function $W(\cdot)$ by

$$
W(u):=V(u)+C_{0} e^{-\int_{t_{0}}^{u} A(v) d v} .
$$

By conditions (3.16), (3.17) and (3.18), we have

$$
\int_{t_{0}}^{t_{n}} e^{-\int_{t_{0}}^{t_{n}} H(v) d v} W(u) d u \leq\left(\alpha+C_{0} L\right)
$$

This yields

$$
e^{-\int_{t_{0}}^{t_{n}} H(v) d v} \int_{t_{0}}^{t_{n}} e^{\int_{t_{0}}^{u} H(v) d v} W(u) d u \leq\left(\alpha+C_{0} L\right)
$$

Then,

$$
\int_{t_{0}}^{t_{n}} e^{\int_{t_{0}}^{u} H(v) d v} W(u) d u \leq\left(\alpha+C_{0} L\right) e^{\mu}
$$

The inequality (3.41) leads to the fact that the sequence

$$
\int_{t_{0}}^{t_{n}} e^{\int_{t_{0}}^{u} H(v) d v} W(u) d u
$$

is bounded, so there exists a convergent subsequence. For brevity, we assume that

$$
\lim _{t \rightarrow \infty} \int_{0}^{t_{n}} e^{\int_{0}^{u} H(v) d v} W(u) d u=\sigma>0 .
$$

Then, we can choose a positive integer $n_{0}$ large enough such that

$$
\int_{t_{n_{0}}}^{t_{n}} e^{\int_{t_{0}}^{u} H(v) d v} W(u) d u<\frac{\delta_{0}}{8 M},
$$

for $n \geq n_{0}$, where $\epsilon>\delta_{0}>0$ satisfies

$$
\begin{aligned}
& \quad\left[\left|\psi\left(t_{n_{0}}\right)\right|+\delta_{0} \sum_{j=1}^{N} \int_{t_{n_{0}}-\tau_{j}\left(t_{n_{0}}\right)}^{t_{n_{0}}}\left|h_{j}(v)\right| d v\right] M \\
& +\left(\left|\dot{x}\left(t_{n_{0}}\right)\right|+\delta_{0} \sum_{j=1}^{N} \frac{\left|b_{j}\left(t_{n_{0}}\right)\right|}{\left|1-\tau_{j}^{\prime}\left(t_{n_{0}}\right)\right|}\right. \\
& \left.\quad+\delta_{0} \int_{t_{n_{0}}-\tau_{j}\left(t_{n_{0}}\right)}^{t_{n_{0}}}\left|C_{j}\left(t_{n_{0}}, v\right)\right| R_{j}(v) d v\right) L \\
& \leq(1-\alpha) .
\end{aligned}
$$


Now, we consider the solution $x(t)=x\left(t, \psi, \dot{x}\left(t_{n_{0}}\right)\right)$ of equation (1.5), for the initial values $\psi$ and $\dot{x}\left(t_{n_{0}}\right)$ such that

$$
\begin{aligned}
\psi\left(t_{n_{0}}\right) & =\frac{3 \delta_{0}}{4}, \quad \dot{x}\left(t_{n_{0}}\right)=\frac{\delta_{0}}{4} \\
|\psi(s)|+|\dot{x}(s)| & \leq \delta_{0}, \quad s \leq t_{n_{0}} .
\end{aligned}
$$

We may choose $\psi$ such that

$$
\psi\left(t_{n_{0}}\right)-\sum_{j=1}^{N} \int_{t_{n_{0}}-\tau_{j}\left(t_{n_{0}}\right)}^{t_{n_{0}}} h_{j}(v) \psi(v) d v \geq \frac{\delta_{0}}{4} .
$$

By a similar argument as in (3.28) and by replacing $\epsilon$ by 1 , this implies that $|x(t)| \leq$ 1. Having in mind the fact that $x$ is a fixed point of $P$, we have, for $n \geq n_{0}$

$$
\begin{aligned}
& \left|x\left(t_{n}\right)-\sum_{j=1}^{N} \int_{t_{n}-\tau_{j}\left(t_{n}\right)}^{t_{n}} h_{j}(v) x(v) d v\right| \\
\geq & \mid e^{-\int_{t_{n_{0}}}^{t_{n}} H(v) d v}\left(\psi\left(t_{n_{0}}\right)-\sum_{j=1}^{N} \int_{t_{n_{0}}-\tau_{j}\left(t_{n_{0}}\right)}^{t_{n_{0}}} h_{j}(v) \psi(v) d v\right) \\
& +\dot{x}\left(t_{n_{0}}\right) \int_{t_{n_{0}}}^{t_{n}} e^{-\int_{u}^{t_{n}} H(v) d v} e^{-\int_{t_{n_{0}}}^{u} A(v) d v} d u \mid \\
& -\left|\int_{t_{n_{0}}}^{t_{n}} e^{-\int_{u}^{t_{n}} H(v) d v} W(u) d u\right| \\
\geq & e^{-\int_{t_{n_{0}}}^{t_{n}} H(v) d v} \frac{\delta_{0}}{4}-\int_{t_{n_{0}}}^{t_{n}} e^{-\int_{u}^{t_{n}} H(v) d v} W(u) d u \\
\geq & e^{-\int_{t_{n_{0}}}^{t_{n}} H(v) d v}\left[\frac{\delta_{0}}{4}-e^{-\int_{0}^{t_{n}} H(v) d v} \int_{t_{n_{0}}}^{t_{n}} e^{\int_{0}^{u} H(v) d v} W(u) d u\right] \\
\geq & \frac{\delta_{0}}{8} e^{-2 \mu}>0 .
\end{aligned}
$$

On the other hand, if the zero solution is asymptotically stable, then $x(t)=$ $x\left(t, \psi, \dot{x}\left(t_{n_{0}}\right)\right) \longrightarrow 0$, as $t \longrightarrow \infty$. It remains only to check that the term

$$
\sum_{j=1}^{N} \int_{t_{n}-\tau_{j}\left(t_{n}\right)}^{t_{n}} h_{j}(v) x(v) d v
$$

decays to zero at infinity to obtain the contradiction. By the mean value theorem 
and condition (3.16), we have

$$
\begin{aligned}
\left|\sum_{j=1}^{N} \int_{t_{n}-\tau_{j}\left(t_{n}\right)}^{t_{n}} h_{j}(v) x(v) d v\right| & =\left|x\left(\eta_{t_{n}}\right)\right|\left|\sum_{j=1}^{N} \int_{t_{n}-\tau_{j}\left(t_{n}\right)}^{t_{n}} h_{j}(v) d v\right| \\
& \leq \alpha\left|x\left(\eta_{t_{n}}\right)\right| \leq\left|x\left(\eta_{t_{n}}\right)\right| .
\end{aligned}
$$

Since $t_{n}$ and $t_{n}-\tau_{j}\left(t_{n}\right) \rightarrow \infty$ as $n \rightarrow \infty$, then also $\eta_{t_{n}} \longrightarrow \infty$. It follows that

$$
\lim _{t_{n} \longrightarrow \infty}\left(x\left(t_{n}\right)-\sum_{j=1}^{N} \int_{t_{n}-\tau_{j}\left(t_{n}\right)}^{t_{n}} h_{j}(v) x(v) d v\right)=0 .
$$

Which leads to a contradiction. This completes the proof of our claim.

In this section, we will give an example to apply our results

Example 3.1. Consider the following nonlinear delay integro-differential equation with variable delay

$$
\ddot{x}+f(t, x, \dot{x}) \dot{x}+\sum_{j=1}^{2} \int_{t-\tau_{j}(t)}^{t} a_{j}(t, s) g_{j}(s, x(s)) d s+\sum_{j=1}^{2} b_{j}(t) x^{\prime}\left(t-\tau_{j}(t)\right)=0,
$$

for $t \geq 0$. We let $A(t):=f(t, x(t), \dot{x}(t))=\frac{1-0.5 \cos (\dot{x}(t) x(t))}{5(t+1)^{\frac{1}{5}}}+2 \tanh t, g_{1}(t, x(t)):=$ $0.2 t \sin x(t), g_{2}(t, x(t)):=0.4 t \cos x(t), a_{1}(t, s)=a_{2}(t, s):=e^{-3(t+s)}, \tau_{1}(t)=0.5 t$, $\tau_{2}(t):=0.5 t$ and $b_{1}(t):=\lambda \frac{0.125 t}{0.25 t^{2}+1}, b_{2}(t):=\sigma \frac{0.125 t}{0.25 t^{2}+1}$ where $0<(\lambda+\sigma) \leq 1 / 7$. Then the zero solution of (3.50) is asymptotically stable.

Proof. We prove that all the hypotheses of Theorem (3.2) hold for equation (3.50). Observe that the conditions (3.14), (3.15) and (3.17) are satisfied, with $R_{1}(t)=$ $R_{2}(t):=t$ and $A_{1}(t):=\frac{0.5}{5(t+1)^{\frac{1}{5}}}+2 \tanh t$, for $t \geq 0$. Now, choose $h_{1}(t):=$ $\lambda \frac{t+1}{t^{2}+1}, h_{2}(t):=\sigma \frac{t+1}{t^{2}+1}$. Clearly, the conditions (3.13) and (3.29) hold. Furthermore, for $t \geq u \geq 0$ we have

$$
\begin{aligned}
\int_{u}^{t} H(v) d v+\int_{0}^{u} A_{1}(t) d v & \geq \int_{0}^{u} A_{1}(t) d v \geq 0.5 \int_{0}^{u} \frac{1}{5(v+1)^{\frac{1}{5}}} d v \\
& \geq \frac{1}{8} u^{\frac{4}{5}}-\frac{1}{8}
\end{aligned}
$$

Consequently, condition (3.18) is satisfied with $a_{0}=1 / 8, b_{0}=-1 / 8$ and $\gamma=4 / 5$.

Remains to prove that the condition (3.16) is also satisfied. There are seven terms on the left hand side of (3.16). By integration we see that,

$$
\begin{aligned}
\int_{0.5 t}^{t} H(v) d v & =\int_{0.5 t}^{t}\left|h_{1}(v)\right| d v+\int_{0.5 t}^{t}\left|h_{2}(v)\right| d v=(\lambda+\sigma) \int_{0.5 t}^{t} \frac{t+1}{t^{2}+1} d v \\
& \leq(\lambda .52)
\end{aligned}
$$


Since $H(t) \geq 0$ for $t \geq 0$, then making use of (3.52) we can derive the estimation

$$
\begin{aligned}
& \int_{0}^{t} e^{-\int_{u}^{t} H(v) d v}|H(u)| \int_{u-\tau_{1}(u)}^{u}\left|h_{1}(v)\right| d v d u \\
\leq & \left(\sup _{u \geq 0} \int_{0.5 u}^{u}\left|h_{1}(v)\right| d v\right) \int_{0}^{t} e^{-\int_{u}^{t} H(v) d v}|H(u)| d v d u \\
\leq & (\lambda+\sigma) 0.84 .
\end{aligned}
$$

The expression of the third term of (3.16) provides the following

$$
\begin{aligned}
& \int_{0}^{t} e^{-\int_{u}^{t} H(v) d v}\left|\left(1-\tau_{1}^{\prime}(u)\right) h_{1}\left(u-\tau_{1}(u)\right)-\frac{b_{1}(u)}{1-\tau_{1}^{\prime}(u)}\right| d u \\
= & \int_{0}^{t} e^{-\int_{u}^{t} H(v) d v}\left|\frac{\lambda 0.25 u+0.5 \lambda}{0.25 u^{2}+1}-\frac{\lambda 0.25 u}{0.25 u^{2}+1}\right| d u \\
= & 0.5 \lambda \int_{0}^{t} \frac{1}{0.25} \frac{1}{u^{2}+4} d u=\lambda \arctan \frac{1}{2} t \leq \lambda \frac{\pi}{2} .
\end{aligned}
$$

and

$$
\begin{aligned}
& \int_{0}^{t} e^{-\int_{u}^{t} H(v) d v}\left|\left(1-\tau_{2}^{\prime}(u)\right) h_{2}\left(u-\tau_{2}(u)\right)-\frac{b_{2}(u)}{1-\tau_{2}^{\prime}(u)}\right| d u \\
= & 0.5 \sigma \int_{0}^{t} \frac{1}{0.25} \frac{1}{u^{2}+4} d u=\sigma \arctan \frac{1}{2} t \leq \sigma \frac{\pi}{2} .
\end{aligned}
$$

For the fourth term of (3.16) we observe that

$$
\begin{aligned}
\left|C_{1}(u, v)\right| & =\left|\int_{u}^{v} a_{1}(w, v) d w\right|=\left|e^{-3 v} \int_{u}^{v} e^{-3 w} d w\right| \\
& =\frac{1}{3}\left|e^{-3 v}\left(e^{-3 u}-e^{-3 v}\right)\right| .
\end{aligned}
$$

But,

$$
\left(e^{-3 v}-e^{-3 u}\right) \geq 0, \text { for } 0 \leq v \leq u
$$

So,

$$
\begin{aligned}
& \int_{0}^{t} e^{-\int_{u}^{t} H(v) d v} \int_{u-\tau_{1}(u)}^{u}\left|C_{1}(u, v)\right| R_{1}(v) d v d u \\
\leq & \int_{0}^{t} \int_{0.5 u}^{u}\left|C_{1}(u, v)\right|\left|R_{1}(v)\right| d v d u \\
\leq & 0.2 \frac{1}{3} \int_{0}^{t} \int_{0.5 u}^{u}\left(e^{-6 v}-e^{-3 v-3 u}\right) v d v d u:=N(t) \\
\leq & 0.00026
\end{aligned}
$$


This is because, the function $N(\cdot)$ is a strictly positive and increasing on $[0, \infty)$ satisfying $N(t) \rightarrow 0.00027$ as $t \longrightarrow \infty$. and

$$
\begin{aligned}
\int_{0}^{t} e^{-\int_{u}^{t} H(v) d v} \int_{u-\tau_{1}(u)}^{u}\left|C_{2}(u, v)\right| R_{2}(v) d v d u & \leq \int_{0}^{t} \int_{0.5 u}^{u}\left|C_{2}(u, v)\right|\left|R_{2}(v)\right| d v d u \\
& \leq 0.00052 .
\end{aligned}
$$

To estimate the fifth term of (3.16), note that

$$
A(t) \leq \frac{1}{5(t+1)^{\frac{1}{5}}}+2 \tanh t \leq 2.3
$$

and

$$
e^{-\int_{s}^{u} A(v) d v} \leq e^{-\int_{s}^{u} A_{1}(v) d v} \leq e^{-2 \int_{s}^{u}\left(\frac{\sinh v}{\cosh v}\right) d v}=\frac{\cosh ^{2} s}{\cosh ^{2} u}
$$

Therefore,

$$
\begin{aligned}
& \int_{0}^{t} e^{-\int_{u}^{t} H(v) d v} \int_{0}^{u} A(s) e^{-\int_{s}^{u} A(v) d v} \int_{s-\tau_{1}(s)}^{s}\left|C_{1}(s, v)\right|\left|R_{1}(v)\right| d v d s d u \\
\leq & (0.2) 2.3 \int_{0}^{t} \int_{0}^{u} e^{-2 \int_{s}^{u} \tanh v d v} \frac{1}{3} \int_{0.5 s}^{s}\left(e^{-6 v}-e^{-3 v-3 s}\right) v d s d v d u \\
\leq & 0.46 \int_{0}^{t} \frac{1}{\cosh ^{2} u} \int_{0}^{u} \frac{1}{3} \frac{1}{4}\left(e^{2 s}+2+e^{-2 s}\right) \\
& \times \int_{0.5 s}^{s}\left(e^{-6 v}-e^{-3 v-3 s}\right) v d s d v d u \\
\leq & \frac{0.46}{12} \sup _{u \geq 0}|\bar{N}(u)| \int_{0}^{t} \frac{1}{\cosh ^{2} u} d u \\
\leq & \frac{0.46}{12} 0.09 \int_{0}^{t} \frac{1}{\cosh ^{2} u} d u=\frac{0.46}{12} 0.09 \tanh t \\
(3.57) \leq & 0.46 \frac{0.09}{12}=0.00345 .
\end{aligned}
$$

This follows from the fact that,

$$
\bar{N}(u):=\int_{0}^{u}\left(e^{2 s}+2+e^{-2 s}\right) \int_{0.5 s}^{s}\left(e^{-6 v}-e^{-3 v-3 s}\right) v d v d s,
$$

is a positive function, increasing on $[0, \infty)$ and $\bar{N}_{1}(u) \leq 0.09$ for any $u \geq 0$.

and

$$
\begin{aligned}
& \int_{0}^{t} e^{-\int_{u}^{t} H(v) d v} \int_{0}^{u} A(s) e^{-\int_{s}^{u} A(v) d v} \int_{s-\tau_{2}(s)}^{s}\left|C_{2}(s, v)\right|\left|R_{2}(v)\right| d v d s d u \\
\leq & 0.0069
\end{aligned}
$$


with $\bar{N}_{2}(u)=\bar{N}_{1}(u)$. Moreover, similar arguments as above show that one can estimate the sixth term of (3.16) as

$$
\begin{aligned}
& \int_{t_{0}}^{t} e^{-\int_{u}^{t} H(v) d v} \int_{0}^{u} e^{-\int_{s}^{u} A(v) d v}\left|C_{1}\left(s, s-\tau_{1}(s)\right)\right| \mid\left(1-\tau^{\prime}(s) \mid\right. \\
& \times\left|R_{1}(s-\tau(s))\right| d s d u \\
\leq & 0.2 \int_{0}^{t} \frac{1}{\cosh ^{2} u} \int_{0}^{u} \frac{1}{4}\left(e^{2 s}+2+e^{-2 s}\right) \frac{0.25}{3}\left(e^{-3 s}-e^{-4.5 s}\right) s d s d u \\
\leq & 0.2 \frac{0.25}{12} \int_{0}^{t} \frac{1}{\cosh ^{2} u} \int_{0}^{u}\left(e^{2 s}+2+e^{-2 s}\right)\left(e^{-3 s}-e^{-4.5 s}\right) s d s d u \\
(3.58) \leq & 0.0041666,
\end{aligned}
$$

and

$$
\begin{aligned}
& \int_{t_{0}}^{t} e^{-\int_{u}^{t} H(v) d v} \int_{0}^{u} e^{-\int_{s}^{u} A(v) d v}\left|C_{2}\left(s, s-\tau_{2}(s)\right)\right| \mid\left(1-\tau^{\prime}(s)|| R_{2}(s-\tau(s)) \mid d s d u\right. \\
\leq & 0.0083332 .
\end{aligned}
$$

For the seventh we have, for $t \geq 0$,

$$
\begin{aligned}
r_{1}(t)= & \frac{\left(b_{1}(t) A(t)+b_{j}^{\prime}(t)\right)}{0.5} \\
& \lambda 0.125\left(\frac{t}{t^{2}+1} A(t)+\frac{1-t^{2}}{\left(t^{2}+1\right)^{2}}\right) \\
= & \lambda 0.125 \frac{1}{t^{2}+1}\left(\frac{0.5 t}{5(t+1)^{\frac{1}{5}}}+2 t \tanh t+\frac{1-t^{2}}{t^{2}+1}\right) \\
\geq & \lambda 0.125\left(\frac{1}{t^{2}+1}\right) .
\end{aligned}
$$

It is clear that the function $r_{1}(\cdot)$ is positive and $H(t) \geq(\lambda+\sigma) \frac{t}{t^{2}+1}$ for $t \geq 0$, which implies that

$$
\begin{aligned}
& \int_{0}^{t} e^{-\int_{u}^{t} H(v) d v} \int_{0}^{u} e^{-\int_{s}^{u} A(v) d v}\left|r_{1}(s)\right| d s d u \\
= & \int_{0}^{t} e^{-\int_{u}^{t} H(v) d v} b_{1}(u) d u=\int_{0}^{t} e^{-\int_{u}^{t} H(v) d v} \frac{\lambda 0.125 u}{0.25 u^{2}+1} d u \\
= & \frac{0.125}{0.25} \frac{\lambda}{\lambda+\sigma} \int_{0}^{t} e^{-\int_{u}^{t} H(v) d v} \frac{(\lambda+\sigma) u}{u^{2}+4} d u \\
(3.60) \leq & \frac{\lambda}{\lambda+\sigma} \frac{0.125}{0.25} \int_{0}^{t} e^{-\int_{u}^{t} \frac{(\lambda+\sigma) v}{v^{2}+1} d v} \frac{(\lambda+\sigma) u}{u^{2}+1} d u \leq \frac{0.125}{0.25}=\frac{\lambda}{\lambda+\sigma} 0.5 .
\end{aligned}
$$


and

$$
\int_{0}^{t} e^{-\int_{u}^{t} H(v) d v} \int_{0}^{u} e^{-\int_{s}^{u} A(v) d v}\left|r_{2}(s)\right| d s d u \leq \frac{\sigma}{\lambda+\sigma} 0.5 .
$$

The summation yields

$$
\begin{aligned}
\alpha: & =(\lambda+\sigma) 0.84+(\lambda+\sigma) 0.84+(\lambda+\sigma) \frac{\pi}{2}+0.00052 .+0.00026 \\
& +0.0083332+0.0041666+\frac{\lambda}{\lambda+\sigma} 0.5+\frac{\sigma}{\lambda+\sigma} 0.5 \\
= & (\lambda+\sigma)\left(1.68+\frac{\pi}{2}\right)+0.51276 \\
\leq & \frac{3.2508}{7}+0.51276=0.97716
\end{aligned}
$$

Also, we remark that the condition (3.19) holds, because $0 \leq 2\left(b_{1}(t)+b_{2}(t)\right)+$ $\frac{1}{12}\left(\bar{N}_{1}(t)+\bar{N}_{2}(t)\right)<+\infty$. Consequently, the zero solution of equation (3.50) is asymptotically stable.

Remark 3.1. It is obvious that by letting $b \equiv 0$ in Example (3.1), we obtain asymptotic stability for the trivial solution of (3.50). Nevertheless, such a conclusion cannot be obtained from the work of $\mathrm{D}$. Pi in [18]. Because, in [18], $R(\cdot)$ is supposed to be bounded while in our consideration is not. Further, the condition (iii) of Theorem 2 in [18] is not applicable here. Thus, our results improve clearly those of [10] and [18].

Acknowledgement. The authors would like to thank the anonymous referee for his/her valuable comments and good advice.

\section{REF E R E N C ES}

1. A. Ardjouni, A. Djoudi, Stability in nonlinear neutral integro-differential equations with variable delay using fixed point theory, J. Appl. Math. Comput. (2014) 44:317-336.

2. A. ARDJouni, A. DJOudi, Fixed points and stability in linear neutral differential equations with variable delays, Nonlinear Analysis 74 (2011) 2062-2070.

3. A. Ardjouni, A. DJoudi, Stability in nonlinear neutral differential with variable delays using fixed point theory, Electronic Journal of Qualitative Theory of Differential Equations 2011, No. 43, 1-11.

4. A. Ardjouni, A. Djoudi, Fixed point and stability in neutral nonlinear differential equations with variable delays, Opuscula Mathematica, VoL. 32, No. 1, 2012, pp. 5-19.

5. A. Ardjouni, A. Djoudi, I. Sounlhia, Stability for linear neutral integrodifferential equations with variable delays, Electron. J. Differ. Equ. Vol. 2012 (2012), No. 172, pp. 1-14.

6. L. C. Becker And T. A. Burton, Stability, fixed points and inverse of delays, Proc. Roy. Soc. Edinburgh 136A (2006) 245-275. 
7. T. A. Burton, Stability by Fixed Point Theory for Functional Differential Equations, Dover Publications, New York, 2006.

8. T. A. Burton, Stability by fixed point theory or Liapunov's theory: A comparison, Fixed Point Theory 4 (2003) 15-32.

9. T. A. Burton, Stability and periodic solutions of ordinary functional differential equations, Academic Press. NY, 1985.

10. T. A. Burton, Fixed points, stability, and exact linearization, Nonlinear Analysis: Theory, Methods \& Applications, Vol. 61, No. 5, pp. 857-870, 2005.

11. A. Djoudi And R. Khemis, Fixed point techniques and stability for neutral nonlinear differential equations with unbounded delays, Georgian Mathematical Journal, Vol. 13 (2006), No. 1, 25-34.

12. J. Faraut, Calcul Intégral, Collection Enseignement SUP-Maths de EDP Sciences, 2006.

13. J. K. HALE, Theory of functional differential equations, Springer Verlag, New York, NY, USA, 1977.

14. C. H. Jin AND J. W. LuO, Stability of an integro-differential equation, Computers and Mathematics with Applications 57 (2009) 1080-1088.

15. J. J. LEVIN AND J. A. NOHEL, Global asymptotic stability for nonlinear systems of differential equations and applications to reactor dynamics, Archive for Rational Mechanics and Analysis, Vol. 5, pp. 194-211, 1960.

16. D. PI, Study the stability of solutions of functional differential equations via fixed points, Nonlinear Analysis: Theory, Methods \& Applications, Vol. 74, no. 2, pp. 639-651, 2011.

17. D. PI, Stability conditions of second order integrodifferential equations with variable delay, Abstract and Applied Analysis, Volume 2014 (2014), Article ID 371639, 11 pages.

18. D. PI, Fixed points and stability of a class of integrodifferential equations, Mathematical Problems in Engineering, Volume 2014 (2014), Article ID 286214, 10 pages.

19. J. L. Schiff, The Laplace Transform: Theory and Applications, Springer-Verlag New York, 1999.

Hocine Gabsi

Faculty of Sciences, Department of Mathematics

University of Annaba

P.O. Box 12

Annaba 23000, Algeria

hocinegabsi@gmail.com

Abdelouaheb Ardjouni

Faculty of Sciences and Technology

Department of Mathematics and Informatics

University of Souk-Ahras 
P.O. Box 1553

Souk-Ahras, 41000, Algeria

abd_ardjouni@yahoo.fr

Ahcene Djoudi

Faculty of Sciences, Department of Mathematics

University of Annaba

P.O. Box 12

Annaba 23000, Algeria

adjoudi@yahoo.com 